\title{
The roles of community health workers in management of non-communicable diseases in an urban township
}

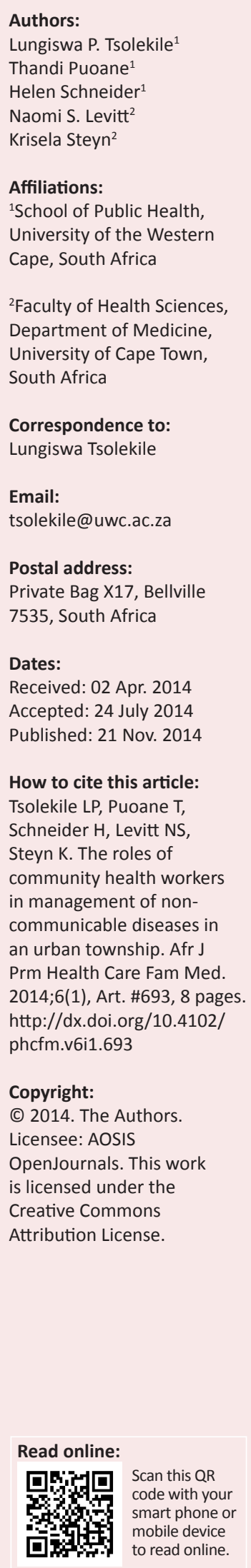

Background: Community health workers (CHWs) are increasingly being recognised as a crucial part of the health workforce in South Africa and other parts of the world. CHWs have taken on a variety of roles, including community empowerment, provision of services and linking communities with health facilities. Their roles are better understood in the areas of maternal and child health and infectious diseases (HIV infection, malaria and tuberculosis).

Aim: This study seeks to explore the current roles of CHWs working with non-communicable diseases (NCDs).

Setting: The study was conducted in an urban township in Cape Town, South Africa.

Method: A qualitative naturalistic research design utilising observations and in-depth interviews with CHWs and their supervisors working in Khayelitsha was used.

Results: CHWs have multiple roles in the care of NCDs. They act as health educators, advisors, rehabilitation workers and support group facilitators. They further screen for complications of illness and assist community members to navigate the health system. These roles are shaped both by expectations of the health system and in response to community needs.

Conclusion: This study indicates the complexities of the roles of CHWs working with NCDs. Understanding the actual roles of CHWs provides insights into not only the competencies required to enable them to fulfil their daily functions, but also the type of training required to fill the present gaps.

Le rôle des agents de santé communautaires dans la gestion des maladies non-contagieuses dans un township urbain.

Contexte: Les agents de santé communautaires (CHW) sont de plus en plus considérés comme un élément capital du personnel de santé en Afrique du Sud et dans d'autres parties du monde. Les CHW assument divers rôles, tels que l'autonomisation des communautés, la prestation de services et le lien entre les communautés et les services de santé. Leurs rôles sont mieux compris dans les domaines de la santé maternelle et infantile et les maladies infectieuses (infection par le VIH, le paludisme et la tuberculose).

Objectif: Cette étude examine les rôles actuels des CHW s'occupant des maladies non contagieuses (NCD).

Cadre: L'étude a été menée dans un township urbain du Cap, en Afrique du Sud.

Méthode: On s'est servi d'une méthode de recherche qualitative et naturaliste qui utilise des observations et des entretiens approfondis avec les CHW et leurs superviseurs travaillant à Khayelitsha.

Résultats: Les CHW ont des rôles multiples pour soigner les NCD. Ils sont éducateurs sanitaires, conseillers, personnel de rééducation et animateurs de groupes de soutien. Ils décèlent les complications de la maladie et aident les membres de la communauté à mieux naviguer dans le système de santé. Ces rôles sont déterminés selon les attentes du système de santé et les réponses aux besoins de la communauté.

Conclusion: Cette étude indique les complexités des rôles des CHW travaillant avec les NCD. La compréhension des rôles des CHW donne une idée non seulement des compétences requises pour leur permettre de s'acquitter de leurs tâches quotidiennes, mais aussi du type de formation requis pour combler les lacunes actuelles.

\section{Introduction}

Community health workers (CHWs) are increasingly being recognised as a crucial part of the health workforce. ${ }^{1}$ In South Africa and worldwide, CHWs have provided health care to communities for many decades and have assumed a variety of roles, including community 
empowerment, provision of services and linking communities with health facilities. Their roles are better understood in the areas of maternal and child health. ${ }^{2}$ For the purposes of this article CHWs are defined as health workers with informal job-related training and no professional or paraprofessional tertiary training, and limited in-service training to contribute to patient management at community level. They may receive a stipend or work voluntarily ${ }^{3}$ and work in communities in which they reside.

$\mathrm{CHWs}^{\prime}$ tasks over the years have evolved from mainly focusing on prevention and promotion to more supportive roles that are associated with the increased burden of chronic lifelong conditions. South Africa, like many countries in transition, has experienced an increase in the burden of chronic lifelong conditions associated with the HIV infection epidemic and the parallel emergence of non-communicable diseases (NCDs). This burden of disease has led to increased workloads, overcrowding at health facilities and poor quality of care, ${ }^{4}$ and has exerted a tremendous strain on human resources in the healthcare system, especially those working at primary healthcare (PHC) level. ${ }^{5}$ The use of $\mathrm{CHWs}$ as part of a solution to the human resources crisis in health settings has been suggested. 6,7

The South African Government has made significant strides in providing free PHC for all, meaning that people with chronic diseases such as NCDs are provided with long-term treatment at no cost and will further need ongoing support to assist them to adhere to treatment. As part of disease management, patients diagnosed with NCDs and placed on treatment are typically advised to attend support groups run or facilitated by professional nurses at the health facilities. ${ }^{6}$ Despite the efforts made at health facilities in managing NCDs, these conditions continue to be managed poorly ${ }^{8}$ and a need for extended and continued care at community level therefore exists.

Given the fact that not all patients utilise health facilities for routine check-ups and that NCDs' symptoms are not recognised until the late stages when complications are apparent, employing CHWs to increase awareness about prevention and control of diseases in communities is crucial. CHWs could strengthen the link between health services and the community, increasing access to services, especially in underserved communities. ${ }^{9}$

In South Africa CHWs have been largely utilised in programmes that target infectious conditions such as HIV/ AIDS and tuberculosis (TB) as well as maternal and child health. Their roles in such programmes are clearly defined and have an established base of evidence illustrating the benefits. $^{2}$ The focus of programmes has been propelled by the need to achieve the Millennium Development Goals, resulting in less attention being given to other conditions such as NCDs. ${ }^{10}$
Numerous interventions for NCDs have been described that utilise the CHW model in disadvantaged communities. ${ }^{11,12}$ Studies from developed countries highlight varied roles and relevance or importance of $\mathrm{CHW}$ in the management of NCDs. These roles include patient education, ${ }^{13,14}$ care and support, ${ }^{13,15,16}$ provision of social support and acting as a liaison with the healthcare system. ${ }^{3}$ For example, in a diabetes intervention CHWs assisted in monitoring blood glucose, blood pressure and potential complications, and provided social support to patients as well as their families. ${ }^{15}$ Despite general support of the CHW model in the management of diseases, there is a need to further understand the roles of CHWs in the prevention and management of NCDs, especially in resource-poor settings.

Recent policy reforms in South Africa prioritise the revitalisation of PHC and stronger community-based services. ${ }^{17}$ On the eve of the re-engineering of PHC and the implementation of PHC outreach teams in South Africa it becomes imperative to understand the roles of CHWs, particularly in caring for conditions previously not included in their work. According to the policy statements on re-engineering of PHC, the work of CHWs has been conceptualised to be comprehensive and to cover household and community level. ${ }^{18}$ With respect to NCDs, at household level their roles will include health promotion, that is education on diet, exercise and lifestyle, and screening for those at high overall cardiovascular disease risk and in particular for diabetes and hypertension, foot care and an integrated approach to adherence support. At community level the focus will be on campaigns, support groups and promoting action on risk factors related to diet and exercise.

\section{Objectives}

In the light of these broadly stipulated roles of $\mathrm{CHWs}$, this study therefore explored $\mathrm{CHWs}^{\prime}$ current roles in NCDs care with a view to identifying existing challenges. The information gathered will assist in informing a training curriculum that relates to $\mathrm{CHWs}^{\prime}$ daily activities and can support a meaningful response to NCDs.

\section{Contribution to field}

Understanding the $\mathrm{CHWs}^{\prime}$ current roles, especially on the eve of re-engineering of $\mathrm{PHC}$, is important for adapting current roles to what will be expected in their new roles in PHC outreach teams. This is especially true as reengineering of PHC may require an expansion of skills as well as new knowledge. Providing an understanding about current roles will give insight into how $\mathrm{CHWs}$ organise their work, the type of activities related to NCDs that they engage in, as well as coping strategies employed in order to address deficiencies in their own work. The results will provide information about the key challenges of managing NCDs at community level, and also highlight possible models that could be used when training opportunities are limited. 


\section{Research methods and design Study design}

A qualitative naturalistic research design utilising observations and in-depth and unstructured interviews to investigate the actual daily activities of $\mathrm{CHWs}$ working with NCD patients was adopted. A naturalistic observation assumes that the roles of CHWs are socially organised and involve examining ${ }^{18}$ the $\mathrm{CHWs}$ in their natural environment whilst carrying out their everyday tasks as they would normally do. ${ }^{19}$ The use of naturalistic observations facilitated an understanding of the complex realities of CHWs working in resource-limited settings with clients with NCDs as well as various influences affecting their work.

\section{Setting}

The non-governmental organisation (NGO) studied was located in Khayelitsha, an urban township in Cape Town, South Africa. Khayelitsha is an economically disadvantaged community with predominantly informal dwellings. According to Census data, Khayelitsha subdistrict has an estimated population of $406779 ; ;^{20}$ however, this number is expected to have increased to over half a million because of an increased influx of people from rural to urban areas over the past few years. ${ }^{21}$ More than half of the population (67\%) is unemployed. ${ }^{22}$

The CHWs who work in this area are employed by an NGO operating in Khayelitsha and receiving funding from the Government. Some of the CHWs within the NGO are also involved in TB programmes, where they act as directly observed TB therapy (short course) supporters. In addition, CHWs also work as home-based carers. At the time of the study there were no ward-based teams operating in the area as is the case in other parts of South Africa.

CHWs within this NGO are supervised by professional nurses and coordinators who oversee their daily activities, including administration. However, the ratio of $\mathrm{CHWs}$ to supervisors varies amongst organisations. The daily clinic nurse workload in the subdistrict is above the national average, as each nurse provides community-based care for about 33 people per day. ${ }^{23}$ Such findings highlight the importance of community-based services as well as the need for CHWs. Compared to other subdistricts in the Western Cape, Khayelitsha has the highest burden of HIV/AIDS, chronic NCDs and injuries. ${ }^{24}$ This makes it an appropriate setting for encouraging preventive and promotive community health services.

\section{Study population and sampling strategy}

Community health workers employed by the NGO were included in the study. Purposive sampling was used in the selection of one NGO, which was chosen on the basis of location as well as its involvement in the management of clients with NCDs. This NGO had 126 employees at the time of the study.

\section{Data collection}

A total of $10 \mathrm{CHWs}$ were observed. Data were collected during August 2011 over a period of four weeks through observations and unstructured interviews. During observations which occurred during a working day (4.5 hours), the researcher collected data through note taking. Unstructured interviews with $\mathrm{CHWs}$ were conducted to stimulate discussions related to roles, issues pertaining to training and understanding of the relations and links with the health facility. These were introduced at the end of each observation when there were questions that stemmed from the observations. Data collected from informal conversations during observation were recorded in the form of notes. This was complemented by the researcher further reflecting on and summarising the proceedings of the day. In order for the researcher to have a clearer understanding of some of the CHW activities and practices observed in the field, in-depth interviews were conducted with two coordinators who supervise CHWs' daily activities.

In preparation for data collection the researcher spent a week with the CHWs, accompanying them in whatever activities they were carrying out. This served three purposes, namely to familiarise the researcher with the day-to-day activities of the NGO, to gain trust and to establish rapport with the CHWs. In addition, the researcher participated in some of the activities undertaken by the CHWs, such as morning prayers and debriefing sessions. Building trust was an important element in the data collection process and assisted in gaining insider status.

\section{Data analysis}

Data from field notes obtained from observations and indepth interviews were analysed through thematic content analysis. The data analysis process started with identification of units of meaning, which were then categorised. Central themes were identified after the data were categorised.

\section{Ethical considerations}

Permission to conduct the study was obtained from the Research and Ethics Committee at the University of the Western Cape, the Department of Health and the NGO which employed the CHWs. Voluntary informed consent was requested from the $\mathrm{CHWs}$ in order for them to participate in the study.

\section{Results}

Analysis of the field notes revealed several primary themes. Principally, the CHWs had multiple roles and these could be summarised into six broad themes: advisor; provision of direct services; monitoring of clients; linking clients with the health system; capacity building; and administration.

The scenario shown in Box 1 indicates some of the roles and realities of CHWs working in the area of NCDs. 
BOX 1: Observation of community health workers' conducting their daily work.

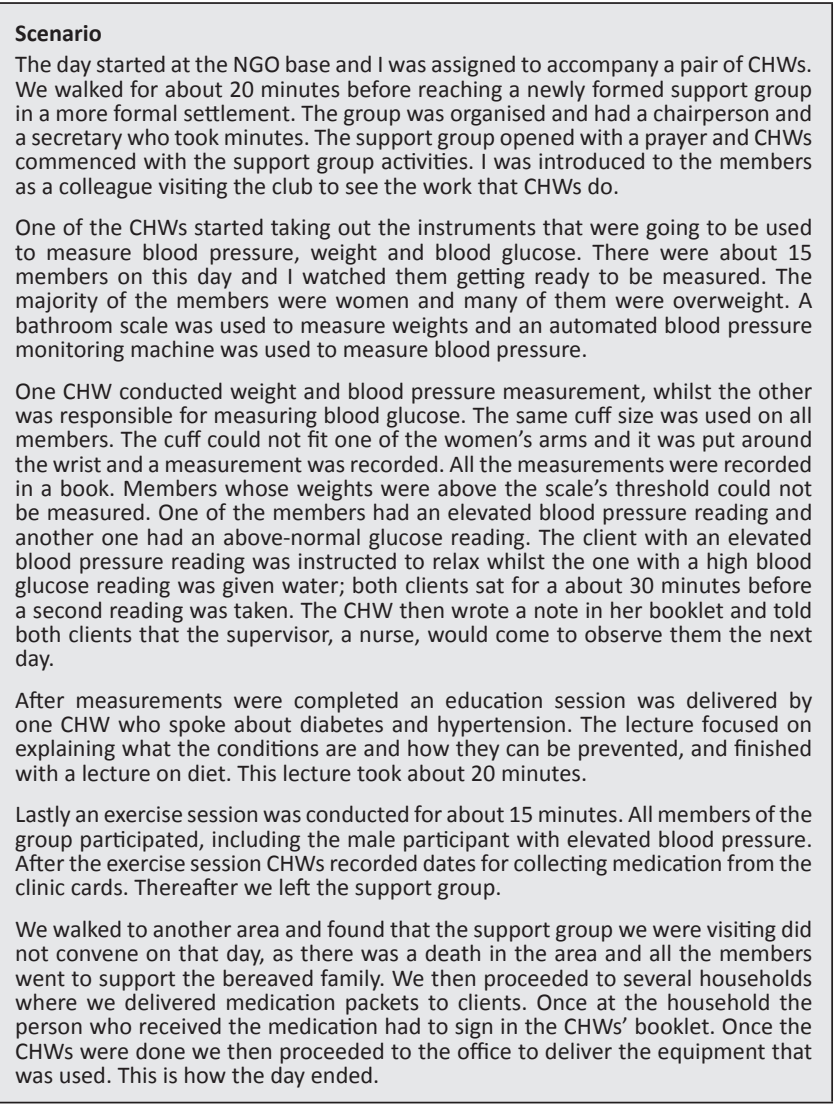

$\mathrm{NGO}$, non-governmental organisation; $\mathrm{CHW}$, community health workers.

\section{Advisor}

CHWs offered advice to clients, which ranged from health advice to social issues that concerned participants. The following field notes recorded during a support group session demonstrate $\mathrm{CHWs}^{\prime}$ advisory role which extends beyond the health domain:

After the health education session at the support group, the $\mathrm{CHW}$ opened the session for questions. One of the elderly participants enquired about the registration process for the Child Support Grant. The CHW could not offer concrete advice but promised to ask relevant people that may have appropriate answers. (Field notes, support group, day 2)

The advisory role was also confirmed by the coordinators during an in-depth interview and one commented as follows: 'Community health workers are there to advise clients on number of issues such as what they should eat and where to go in order to get help' (Co-ordinator, day 8).

\section{Provision of direct services}

It was evident from the observations that $\mathrm{CHWs}$ provided direct services to their clients which included facilitation of support groups, health education, distribution of medication as well as rehabilitation exercises.

\section{Facilitation of support groups}

Facilitation of support groups consisting of clients with diabetes and/or hypertension was one of the roles identified. As facilitators, CHWs assumed a leadership position:

On arrival at the support group the participants were already sitting and waiting for the CHW. She introduced herself and then explained how the session will be structured. Thereafter she started with the day's activities. (Field notes, support group, day 1)

This was also observed in three other support groups that were facilitated by $\mathrm{CHWs}$, where $\mathrm{CHWs}$ led support group sessions.

\section{Health education}

At the support groups and within households, CHWs work as educators. Education sessions offered by CHWs varied and included matters pertaining to nutrition in the management of NCDs as well as explaining about risk factors, symptoms and prevention measures for diabetes and hypertension. In certain instances CHWs explained healthy eating as consuming plenty of fruit and vegetables. The observation highlights gaps in knowledge on diet and nutrition:

I attended an education session delivered by a CHW at a support group. She started the session by describing hypertension; she further explained the risk factors for hypertension and how conditions such as hypertension can be prevented. She completed the session with a lecture on nutrition and how nutrition can prevent diabetes and hypertension. The CHW emphasised the importance of a 'healthy diet' and explained that this diet should consist of vegetables and fruit. The information provided to the participants was in the form of a didactic lecture with no illustrations or educational materials to assist the participants in their learning and the $\mathrm{CHW}$ in her teaching. (Field notes, support group, day 3 )

In addition, health education also occurred during home visits; however, the health education at homes was not of the same depth as within support groups. Health education provided during home visits was in response to questions posed by a particular client rather than routine practice as done in support groups.

\section{Distribution of medication}

Chronic medication for selected clients assigned to CHWs was distributed in households and at the designated support groups. The delivery of medication to clients by CHWs is a way of improving access to treatment and serves a dual purpose for both the clients and the health facility, by not only ensuring that clients receive medication timeously, but also enabling them to bypass the long queues and long waiting times usually encountered at health facilities and the transport costs involved:

After recording the medication in the record book, we went to deliver the medication to clients. We first visited a client that was bedridden to deliver her medication; we then visited a support group where we delivered the bulk of the medication. All the clients collected their medication and were then reminded about their next doctor's appointment. (Field notes, support group, day 6) 
In certain instances $\mathrm{CHW}$ did emphasise the importance of taking medication as prescribed; however, this was not a common practice and was mostly communicated during household visits.

Although education about medication was not included in the sessions with clients, CHWs still assisted with medication-related issues and communicated these to the health facility or nurse supervisor. This is highlighted in the scenario and conversation about what $\mathrm{CHW}$ do when confronted with issues relating to medication:

When CHWs delivered medication at a client's house, the client could not recognise some of the medication that was included in the package and relayed his concern to the CHW. (Field notes, home visit, day 8)

The CHW resolved this issue through a verification process:

\begin{abstract}
'I look at the boxes, write down the substances, the milligrams [dosage] and the drug name that is written in the box. I then go to the book where we record the medication to see if what is given corresponds with what is in the book. If I'm not sure, I ask the nurse supervisor or pharmacist at the clinic. After getting a response I then tell the client what I have been told.' (Conversation with a CHW, support group, day 6)
\end{abstract}

\section{Rehabilitation services}

A single CHW was observed providing exercises to a client with hemiplegia resulting from a stroke. The CHW was assisted by another CHW to handle the client. Whilst conducting the exercises the CHW kept communicating with the client, who seemed pleased with the service provided. The CHW then encouraged the client to visit the local health facility in order to access further rehabilitation services. Interestingly, no clients from the other support groups were observed receiving rehabilitation exercises. The lack of CHWs assisting clients with rehabilitation exercises in other support groups was explained by the co-ordinator:

'A few years ago we received funding to train CHWs to work with community members that could not readily access physiotherapy services. Our CHWs were then trained in rehabilitation exercise; however, when the funding ended we could not continue with the work we were doing. Currently we only have two CHWs who were part of that training and others have retired.' (Co-ordinator, day 7)

\section{Monitoring of clients}

CHWs assisted support group members with information that would enable them to better manage their conditions. Whilst conducting support groups, the CHWs collected anthropometric measurements, namely blood pressure, blood glucose and weights of all the participants. Blood pressure was measured using only one cuff size, despite varying mid-upper arm circumferences. Notably, in cases where the cuff was too small to fit the size of upper arm, CHWs placed the cuff on a client's wrist. Finger-prick blood glucose was measured using a new lancet for each person, and clients' readings were recorded. Weights were measured using a dial bathroom scale and put on surfaces that were sometimes uneven. During weight measurements at no point was the scale calibrated. Calculations of body mass index were not performed. In all the observations, individuals with abnormal blood pressure and/or blood glucose readings were informed of their results and referred to the facility. The appreciation on the faces of the support group members was clearly evident during the taking of measurements. During home visits no CHWs were observed taking any client measurements.

\section{Linking clients with the health system}

At the support groups CHWs referred people who were identified with elevated blood glucose levels as well as raised blood pressure to the nurse supervisor, who then referred the clients to the nearest health facilities. Referred clients were given a referral letter by the nurse to present to the health facility. Although clients reported being attended to at the health facility, no letters were sent back to the nurse supervisor or CHW. When clients were followed up by $\mathrm{CHWs}$ through home visits, $\mathrm{CHWs}$ could only rely on the clients' recollection of their clinic attendance and by inspecting the clinic card to ensure that the client did indeed visit the health facility as instructed.

\section{Capacity building Peer educator}

CHWs assisted other CHWs with 'on-the-job' experience. It was evident from one of the field observations that $\mathrm{CHWs}$ also acted as peer educators by assisting peers with the skills required to fulfil daily tasks:

On one of the visits I noticed that $\mathrm{CHWs}$ were working as a trio and one CHW would constantly be observing what was happening. When I enquired about this, I was told that the third CHW was newly recruited and therefore did not have experience in doing the work, thus they had to teach her what is usually done. (Field notes, support group, day 9)

According to the coordinators, this was a way of infusing knowledge about field work to new recruits and to assist them in gaining on-the-job experience. One of the coordinators commented as follows:

'We have team leaders that we use in the field; they are the ones that we use to partner with a newly recruited CHW. [...] the team leaders are CHWs who have been working for the organisation for a long time and they really know the work.' (Co-ordinator, day 7)

\section{Administration}

CHWs completed forms with information relating to the clients on a daily basis. The information recorded includes patients' medication, and particulars of the clients to be visited on that day. In addition, CHWs completed daily statistic sheets for clients (clients seen the day before or planned to be seen). The completion of forms was a daily activity done at the beginning of the day, whilst some of the forms that involved updating patient information were completed after home visits and submitted to the 
coordinators on the next day. CHWs were also responsible for collected signatures of clients when they receive medication, so as to keep a record of the clients who have collected their medication.

\section{Discussion}

The findings of this study show that CHWs fulfil a variety of roles in the management of NCDs. As such they have a crucial role in community level NCD public health care in South Africa. It is evident that the varied roles fulfilled by $\mathrm{CHWs}$ seem to be in response to clients' needs. Consequently a degree of flexibility in the way they approach their work may be necessary so as to allow them to react to the community's needs.

In a society where community needs vary, acting as an advisor may be one of the crucial roles played by CHWs. However, as demonstrated in this study, their knowledge and available support material may need to extend beyond health-related issues, especially in communities with social problems. Other studies have shown that $\mathrm{CHWs}$ trained in health-related issues such as diabetes management were often confronted with issues unrelated to diabetes, and therefore suggested the need to prepare them for unanticipated non-diabetes issues. ${ }^{11}$ Such expectations pose a challenge to CHWs' scope of practice and what can be realistically expected of them. In addition, this illustrates the need to form meaningful networks with other community structures such as social development. These findings further support the broader debate that CHWs need to be integrated into Government programmes and be incorporated into professional teams ${ }^{1}$ who can offer support. Furthermore, communication with other team members whilst in the field can provide more streamlined services for the clients.

Playing the role of a health educator meant that CHWs are expected to act as sources of information, creating an expectation that $\mathrm{CHWs}$ possess current and relevant information related to NCDs. The study also highlighted the shortfalls in CHWs' knowledge, in that information provided was not always accurate. Inability to clarify certain nutrition concepts emphasises the need for training and continuous education that is tailored to the context that addresses the everyday realities of CHWs, as well as the need for teaching aids to further enforce and standardise the health information given to clients. Indeed it has been suggested that the community's perception of CHW knowledge, skills and ability to assist communities with their health needs is crucial in inspiring respect and acceptance of CHW services. ${ }^{9}$

As lay workers based in communities, $\mathrm{CHWs}$ play a fundamental role in community-based support groups, and as demonstrated in this study, within such groups their roles could vary. As facilitators, $\mathrm{CHWs}$ require a diverse range of skills which includes communication skills, as part of facilitation includes communicating with the audience. CHWs set the subject or topics for discussion and have the potential to influence the focus of the group, and as such require leadership skills. This leadership role presents its own challenges and expectations, as it would imply that CHWs are expected to know how to guide and support group members. In addition, increased demands to support and provide information may rise to a point where expectations on the leader may exceed their capabilities. ${ }^{25}$ Thus it is necessary to structure facilitation of support groups in such a way that forges partnerships, and co-ownership between participants and facilitators.

The distribution of medication to clients on chronic medication serves several purposes that benefit the clients as well as the health facility. Distribution of medication in the community may assist in improving access to treatment, ensure that medication is received timeously and reduce long waiting times in pharmacies at the health facilities. Clearly delivering medication in such settings is helpful, as it minimises the number of trips to the health facility and also reduces overcrowding at health facilities. This benefit could be enhanced considerably if CHWs were to advise on improving adherence and provide education relating to medication for the management of conditions. Our findings highlight the lack of adherence support given to clients with NCDs, in stark contrast to the extensive support provided by CHWs to patients on HIV infection and TB treatment.

As CHWs in this study mainly worked with individuals already known to have a chronic disease, it is crucial that they possess skills in physical rehabilitation of clients. This is particularly pertinent because of the increase in risk factors for cardiovascular disease; an increase in the aging population suggests a similar increase in the burden of cardiovascular disease in sub-Saharan Africa and elsewhere in the developing world. In the absence of appropriate interventions, stroke and heart disease-related deaths are expected to increase from 3 million in 1998 to 5 million in 2020 in developing regions as outlined by the World Health Organization. ${ }^{26,27}$ Such statistics suggest that there will be a need for community-based services such as CHWs with specialised skills to deal with the burden of increased numbers of people with physical disabilities caused by strokes and those who suffer from ischaemic heart disease. This will then extend services to clients in areas where there is a scarcity of rehabilitation workers or where people may have limited access to these services.

The role of CHWs in measuring vital parameters in children has been shown to be feasible (reliable) in numerous studies on children. ${ }^{28}$ This role could be extended to NCDs, for the detection of abnormal readings and thus the facilitation of referral. In instances when such measurements are incorrectly conducted, this may lead to $\mathrm{CHWs}^{\prime}$ work being devalued. Furthermore, such inadequacies in practice highlight insufficiencies in training and supervision. Thus adequate training, supervision and regular evaluation of tasks are essential in ensuring good practice.

One of the major roles for CHWs has been to connect or link community members to the health facility. ${ }^{7}$ In this 
study this role was fulfilled in numerous ways, such as the delivery of medication and referral of clients identified in the community to a health facility. In communities where health-seeking behaviour is poor and people only visit health facilities when they have serious symptoms, $\mathrm{CHWs}$ can play a crucial role in identifying problem cases early enough for health professionals to intervene timeously. However, it is evident that the relationship or communication between health facilities and $\mathrm{CHWs}$ has its challenges. Improved communication between health facilities and CHWs could assist in increasing $\mathrm{CHWs}^{\prime}$ legitimacy in the eyes of the community.

The role of CHWs as peer educators is an illustration of innovative thinking by their NGO in order to deal with a lack of appropriate formal training services, although innovative peer education needs to be supervised and better formalised to ensure that the knowledge transferred is appropriate and accurate. Peer education in this context shows how an organisation tailors practices to meet the needs of those they serve.

This article shows some of the limitations and challenges to the roles and responsibilities of $\mathrm{CHWs}$ in an NCDs programme. For example, home visits were not utilised maximally, as these could serve as places where health education is delivered. In addition, the work of CHWs concentrated on people with NCDs, thus excluding the population at risk which could benefit from their services. In a place where there is an increase in NCDs there is a need for primary prevention, and $\mathrm{CHWs}$ can be used in communities to identify those at risk.

\section{Limitations of the study}

The study is limited by the methodology utilised. Although observations are an accepted qualitative research method, they have several constraints, one being that observations are context-specific. A second constraint is that study subjects may alter behaviour when observed by an outsider. Furthermore, observations were based on data collected from one NGO. It is thus important to remember that different organisations may organise work differently. Therefore the findings of this study indicate the issues relating to roles in managing NCDs rather than being generalisable.

\section{Recommendations}

On the basis of the findings of this study, the following recommendations are made:

1. CHWs should be empowered with resources as well as forging links with non-health organisations so that they can better refer clients with non-health problems.

2. The main issue of this study is the role of $\mathrm{CHW}$ s relating to NCDs. On the one hand there are factors that may inhibit their roles, which may undermine their value in society; on the other hand, the varied roles add value to their work. There is therefore a need to develop the capacity of CHWs in a sustained manner and to create on-going education that is organised and relevant to the context in which they work. In addition, it will be crucial to explore the concept of peer education and how the process can be formalised and supported.

3. It is important to look at community-to-facility referral pathways, as this is important in linking clients to formal health services as well as in the continuity of care, especially for chronic conditions.

\section{Conclusion}

This study shows the complexities of the work done by CHWs working with NCD-related conditions. Thus, understanding the actual roles of $\mathrm{CHWs}$ provides insights not only into the competencies required to enable them to fulfil their daily functions, but also into the type of training required to fill the present gaps.

\section{Acknowledgements}

We would like to acknowledge the Chronic Diseases Initiative for Africa for the funding support. We would like to thank the participants and the NGO concerned for their time and valuable contributions during the study.

\section{Competing interests}

The authors declare that they have no financial or personal relationship(s) that may have inappropriately influenced them in writing this article.

\section{Authors' contributions}

L.P.T. (University of the Western Cape) initiated the study and was responsible for the data collection, data analysis and writing of the manuscript. T.P. (UWC) contributed in conceptualisation of the study design, preparation of the manuscript and critical revision of the article. H.S. (UWC) assisted in conceptualisation of the study design, preparation of the manuscript and critical revision of the article. N.L. (University of Cape Town and Chronic Disease Initiative for Africa) contributed in conceptualisation of the study, drafting the manuscript and critical revision of the article. K.S. (Chronic Disease Initiative for Africa) contributed in conceptualisation of the study, drafting the manuscript and critical revision of the article.

\section{References}

1. Lehmann U, Sanders D. Community health workers: What do we know about them? The state of the evidence on programmes, activities, costs and impact on health outcomes of using community health workers. Geneva: World Health Organization; 2007, pp. 1-42.

2. Lewin S, Munabi-Babigumira S, Glenton C, Daniels K, Bosch-Capblanch X, Van Wyk BE, Odgaard-Jensen J, Johansen M, Aja GN, Zwarenstein M, Scheel IB. Lay health workers in primary and community health care for maternal and child health and the management of infectious diseases. Cochrane Database Syst Rev. 2010 Mar 17;(3):CD004015.

3. Lewin SA, Babigumira SM, Bosch-Capblanch, et al. Lay health workers in primary and community health care: A systematic review of trials. Geneva: World Health Organization [homepage on the Internet] c2006 [cited 2014 Jan 11]. Available from: www.who.int/rpc/meetings/LHW_review2.pdf

4. Mayosi BM, Flisher AJ, Lalloo UG, Sitas F, Tollman SM, Bradshaw D. The burden of non-communicable diseases in South Africa. Lancet 2009; 374(9693):934-947. http://dx.doi.org/10.1016/S0140-6736(09)61087-4 
5. Tollman SM, Kahn K, Sartorius B, Collinson MA, Clark SJ, Garenne ML. Implications of mortality transition for primary health care in rural South Africa: A population-
based surveillance study. Lancet 2008; 372(9642):893-901. http://dx.doi. based surveillance study. Lancet 200
org/10.1016/S0140-6736(08)61399-9

6 . Steyn K, Levitt NS. Health services research in South Africa for chronic diseases of lifestyle. In: Steyn K, Fourie J, Temple N, editors. Chronic diseases of lifestyle in South Africa: 1995-2005, Technical report. Cape Town: South African Medical Research Council; 2006, pp. 226-248.

7. Singh P. One million community health workers: technical task force report [homepage on the Internet] c2011 [cited 2014 January 11]. Available from: http://1millionhealthworkers.org/files/2013/01/1mCHW TechnicalTaskForceReport.pdf

8. Steyn K, Levitt D, Patel M, et al. Hypertension and diabetes: Poor care for patients at community health centres. S Afr Med J. 2008;98(8):618-622.

9. Jaskiewicz W, Tulenko K. Increasing community health worker productivity and effectiveness: A review of the influence of the work environment. Hum Resour Health 2012;10(1):38. http://dx.doi.org/10.1186/1478-4491-10-38

10. Perry $H$, Zuliger R. How effective are community health workers? An overview of current evidence with recommendations for strengthening community health worker programs to accelerate progress in achieving the health-related Millennium Development Goals [homepage on the Internet] c 2012 [cited 2014 Jan 15]. Available from: http://www.coregroup.org/storage/Program_Learning/ Community_Health_Workers/review\%20of\%20chw\%20effectiveness\%20for\%20 mdgs-sept2012-condensedversion.pdf

11. Puoane T, Tsolekile L, Sanders D, Parker W. Chronic Non-communicable diseases. In: Barron P, Roma-Reardon J, editors. South African Health Review 2008. Durban Health Systems Trust, 2008; pp. 73-87.

12. Wadler BM, Judge CM, Prout M, Allen JD, Geller AC. Improving breast cancer control via the use of community health workers in South Africa: A critical review. J Oncol. 2011; Article ID 150423, 8 pages.

13. Gary TL, Bone LR, Hill MN, Levine DM, McGuire M, Saudek C, Brancati FL. Randomized controlled trial of the effects of nurse case manager and community health worker interventions on risk factors for diabetes-related complications in urban African Americans. Prev Med. 2003;37(1):23-32. http://dx.doi. org/10.1016/S0091-7435(03)00040-9

14. Cherrington A, Ayala GX, Amick H, Allison J, Corbie-Smith G, Scarinci I. Implementing the community health worker model within diabetes management
challenges and lessons learned from programs across the United States. Diabetes challenges and lessons learned from programs across the United States. Diabet
Educator. 2008;34(5):824-833. http://dx.doi.org/10.1177/0145721708323643

15. Fedder DO, Chang RJ, Curry S, Nichols, G. The effectiveness of a community health worker outreach program on healthcare utilization of west Baltimore City Medicaid patients with diabetes, with or without hypertension. Ethn Dis. 2003;13(1):146
16. McDermott R, Tulip F, Schmidt B, Sinha A. Sustaining better diabetes care in remote indigenous Australian communities. BMJ 2003;327(7412):428. http:// dx.doi.org/10.1136/bmj.327.7412.428

1 7. Naledi T, Barron P, Schneider H. Primary health care in SA since 1994 and implications of the new vision for PHC re-engineering. In: Padarath A, English $\mathrm{R}$ editors. South African health review. Durban: Health Systems Trust, 2011; pp. $17-28$.

18 . Bolster D, Manias E. Person-centred interactions between nurses and patients during medication activities in an acute hospital setting: Qualitative observation and interview study. Int J Nurs Stud 2010;47(2),154-165. http://dx.doi. org/10.1016/j.ijnurstu.2009.05.021

19. Pillay $Y$, Barron P. The implementation of PHC re-engineering in South Africa. Public Health Association of South Africa [homepage on the Internet] c2011 [cited 2013 July 20]. Available from: http://www.phasa.org.za/articles/theimplementationof-phc-re-engineering-in-south-africa.html

20 . Department of Social Services and Poverty Alleviation. The population register update: Khayelitsha [homepage on the Internet] c2006, p. 72 [cited 2013 July 20]. october_2007_publish_date.pdf

2 1. Provincial and Local Government. Nodal economic profiling project: Khayelitsha Western Cape. Republic of South Africa [homepage on the Internet] c2011, p. 8 [cited 2013 July 20]. Available from: http://www.btrust.org.za/library/assets/ uploads/documents/3 CIPPN Khayelitsha\%20profile.pdf

2 2. Department of Social Services. Department of Social Development nodal baseline survey: Khayelitsha results [homepage on the Internet] c2010 [cited 2013 July 20]. Available from: http://www.sarpn.org/documents/d0003004/

2 3. Day C, Monticelli F, Barron P, Haynes R, Smith J, Sello E, editors. The District Health Barometer 2008/09. Durban: Health Systems Trust; 2010.

2 4. Groenewald P, Bradshaw D, Daniels J, et al. Local-level mortality surveillance in resource-limited settings: A case study of Cape Town highlights disparities
in health. Bull WHO 2010; 88(6): 444-451. http://dx.doi.org/10.2471/ BLT.09.069435

2 5. Keene A. Complexity theory: The changing role of leadership. Ind Comm Training 2000;32(1):15-18. http://dx.doi.org/10.1108/00197850010311121

2 6. Leeder S, Raymond S, Greenberg H, Liu H, Esson K. A race against time: The challenge of cardiovascular disease in developing countries. New York: Centre for Global Health and Economic Development, Columbia University; 2004.

2 7. World Health Organization. Report of the Commission on Macroeconomics and Health: Investing in health for economic development. Geneva: World Health Organization; 2001.

2 8. Amarchandi R, Sharmaii $H$, Krishnaniii A. Community health workers can be trained to measure blood pressure: Experience from India. Regional Health Forum. 2013;17(1):26-31. 